\title{
SOME REMARKS ON THE MEAN VALUE OF THE RIEMANN ZETA-FUNCTION AND OTHER DIRICHLET SERIES. III
}

\author{
K. RAMACHANDRA \\ In honour of Srimathi and Sri S. Ramanujan
}

\section{Introduction}

As in the previous papers with the same title, the subject matter of the present paper is the study of the mean value

$$
M(\sigma)=M(F, \sigma, l, k, T, H)=\frac{1}{H} \int_{T}^{T+H}\left|\frac{d^{l}}{d s^{l}}\left((F(s))^{k}\right)\right|_{s=\sigma+i t} d t, \quad(\sigma \geqq 1 / 2),
$$

where $F(s)$ and $(F(s))^{k / 2}$ are Dirichlet series convergent in $\sigma>1$. In addition $F(s)$ is assumed to admit an analytic continuation in $\sigma \geqq 1 / 2, T \leqq t \leqq T+H$, and there $|F(s)| \leqq \exp \left((\log T)^{100}\right)$ (the constant 100 is unimportant and can be replaced by bigger ones if necessary), $10 \leqq(\log T)^{1 / m} \leqq H \leqq T, l \geqq 0, m \geqq 1$, being integer constants and $k$ a real constant. Of course by $(F(s))^{k}$ we mean the analytic continuation, of the series for $(F(s))^{k}$ convergent in $\sigma>1$, to $\sigma \geqq 1 / 2$ on all possible lines parallel to the real axis. Writing

$$
(F(s))^{k / 2}=\sum_{n=1}^{\infty} \frac{a_{n}}{n^{s}}, \quad(\sigma>1),
$$

and assuming some fairly general conditions on the sequence $\left\{a_{n}\right\}$ of complex numbers we proved in the previous paper I (see [1]) that

$$
M(1 / 2)>\left(\sum_{n \leqq H} \frac{\left|a_{n}\right|^{2}}{n}\right)(\log H)^{l}(\log \log H)^{-C_{k, l}^{\prime},} \quad\left(C_{k, l}^{\prime}>0\right),
$$

where $C_{k, l}^{\prime}$ is a constant and $T$ exceeds a constant depending on $k, l$ and $m$. (We proved this under the assumption like $|F(s)| \leqq T^{100}$, but it is easy to moderate this condition into conditions like $\left.|F(s)| \leqq \exp \left((\log T)^{100}\right)\right)$. In II (see [2]) we assumed that $k=1$ without altering the other conditions and the outcome was

$$
M(1 / 2) \gg\left(\sum_{n \leqq H} \frac{\left|a_{n}\right|^{2}}{n}\right)(\log H)^{l}
$$

doi:10.5186/aasfm.1980.0522 
where $\gg$ is the familiar Vinogradov symbol.

As a special case of the results of II we can quote

Theorem 1. If $k$ and $m$ are positive integers and $l$ a non-negative integer, then for $10 \leqq(\log T)^{\mathbf{1} / m} \leqq H \leqq T$,

$$
\frac{1}{H} \int_{T}^{T+H}\left|\frac{d^{l}}{d s^{l}}\left((\zeta(s))^{k}\right)\right|_{s=1 / 2+i t} d t>C_{k, l}(\log H)^{k^{2 / 4+l}},
$$

where $C_{k, l}>0$ depends only on $k$ and $l$ and $T$ exceeds a positive constant depending on $k, l$, and $m$. If, on the other hand, $k$ is a positive real constant, the theorem is still true under the assumption $0 \leqq l \leqq k$ and $(\zeta(s))^{k / 2}$ is analytic in $\sigma>1 / 2, T \leqq t \leqq T+H$. All these results hold under the respective assumptions when in the integral on the left $s=1 / 2+i t$ is replaced by $s=\sigma+i t$ with $1 / 2<\sigma<1 / 2+O(1 / \log H)$. Further, if we write $s=1 / 2+(1 / \log H)+i t$ in the integral, then $k$ can be any real constant and $l$ any non-negative integer, provided we assume that $(\zeta(s))^{k / 2}$ is analytic in $\sigma>1 / 2$.

The present paper deals with upper bounds for the mean value. As is to be expected the restrictions on $F$ and $H$ have to be very heavy. As a first restriction, we impose $H=T^{\lambda}$, where $0<\lambda<1$, and $\lambda$ is a constant, in what follows. Before proceeding further with general theorems we quote two samples which follow as immediate corollaries to our general theorem.

Theorem 2. Let $l$ be a non-negative integer constant and $H=T^{\lambda}$, where $\lambda$ is a constant satisfying $1 / 2<\lambda<1$. Then

$$
\frac{1}{H} \int_{T}^{T+H}\left|\zeta^{(l)}(1 / 2+i t)\right| d t=O\left((\log T)^{1 / 4+l}\right),
$$

where the $O$-constant is independent of $T$.

Theorem 3. Let $(\zeta(s))^{k / 2}$ be analytic in $\sigma>1 / 2, t \geqq 20$, where $k$ is a positive constant satisfying $0<k<4$. Let $l$ be a non-negative integer constant and $\lambda$ a real constant satisfying $0 \leqq l \leqq k / 2$ and $k / 4<\lambda<1$. Then with $H=T^{\lambda}$ we have

$$
\frac{1}{H} \int_{T}^{T+H}\left|\frac{d^{l}}{d s^{l}}\left((\zeta(s))^{k}\right)\right|_{s=1 / 2+i t} d t=O\left((\log T)^{k^{2} / 4+l}\right),
$$

where the $O$-constant is independent of $T$. If we set $s=1 / 2+1 / \log T+i$ instead of $s=1 / 2+i t$ in the integral, and assume that $(\zeta(s))^{k / 2}$ is analytic in $\sigma>1 / 2$, then the inequality mentioned above holds (without the restriction $0 \leqq l \leqq k / 2$ ) for all integers $l \geqq 0$.

Remark 1. All the constants in these theorems and also all others mentioned in this paper are effective. 
Remark 2. These theorems are valid when $\zeta(s)$ is replaced by the $L$-series $L(s, \chi)$, where $\chi$ is a residue class character, and it is also possible to work out the hybrid analogues of these theorems.

Theorems more general than 2 and 3 will be found below in the paper. The general plan of the paper is as follows. Part A deals with two general theorems on mean values. Part B deals with a theorem which gives Theorem 3 as a corollary. Part $\mathrm{C}$ deals with a theorem which gives Theorem 2 as a corollary. We will have occasions to use theorem

$$
\int_{0}^{H}\left|\sum_{n=1}^{\infty} a_{n} n^{i t}\right|^{2} d t=\sum_{n=1}^{\infty}(H+O(n))\left|a_{n}\right|^{2},
$$

due to H. L. Montgomery and R. C. Vaughan. For a simple proof of this version see [3].

Acknowledgement. The author is indebted to Professor M. Jutila for much encouragement and in particular for correcting a number of oversights in the first draft.

\section{Part A}

Theorem 4. Let $G(s)=\sum_{n=1}^{\infty} b_{n} / n^{s}$ be absolutely convergent for $\sigma>1 \quad\left(b_{n}\right.$ are complex numbers and $s=\sigma+i t)$ and suppose $G(s)$ admits an analytic continuation (which we once again denote by $G(s)$ ) in $\sigma>1 / 2, T-H / 2 \leqq t \leqq T+3 H / 2$, where $10 \leqq(\log T)^{1 / m} \leqq H \leqq T$ and $m$ is a positive integer constant. Suppose that in the region $\sigma \geqq 1 / 2, T-H / 2 \leqq t \leqq T+3 H / 2, \quad G(s)$ is defined and continuous and satisfies $|G(s)| \leqq$ $\exp \left((\log T)^{100}\right)$. For $\sigma \geqq 1 / 2$ put

$$
M_{1}(\sigma)=\frac{1}{H} \int_{T}^{T+H_{1}}\left|G(s)-\sum_{n \leqq H} \frac{b_{n}}{n^{s}}\right|^{2} d t, \quad\left(H_{1}>0\right),
$$

and define $\psi\left(r, T, H_{1}\right)=M_{1}(1 / 2+r) X^{r}$ for some $X \geqq 1$ and all $r \geqq 0$. Then under the assumption $X \leqq H^{2-\delta}$ for any fixed constant $\delta>0$, we have for $0<r<r^{\prime}=O(1)$ and $0<\eta<1$ the estimation

$$
\begin{aligned}
\psi(r, T, H)= & O\left(\psi\left(0, T-H^{\eta}, H+2 H^{\eta}\right)\left(\log \left(\frac{r^{\prime}}{r}\right)\right)\right) \\
& +O\left(\psi\left(r, T-H^{\eta}, H+2 H^{\eta}\right)\left(\log \left(\frac{1}{r^{\prime}-r}\right)\right) H^{-\delta_{1}\left(r^{\prime}-r\right)}\right),
\end{aligned}
$$

where the $O$-constants depend only on $\eta, \delta$ and the bound for $r^{\prime}$ and not on $T, H, r, r^{\prime}$, and $\delta_{1}$ depends only on $\delta$ and is positive. 
Remark. A similar theorem is true if in place of $\left|G(s)-\sum_{n \leqq H} b_{n} / n^{s}\right|^{2}$ we consider for instance $\left|G(s)-\left(\sum_{n \leqq H} d_{n}^{\prime} / n^{s}\right)^{2}\right|$, where $\sum_{n=1}^{\infty} d_{n}^{\prime} / n^{s}$ is convergent for at least one value of $s$ to $\sqrt{G(s)}$. However, corresponding to these changes we change the condition on $X$ accordingly. For example in the last case mentioned just now we have to limit $X$ by $1 \leqq X \leqq H^{1-\delta}$ in place of $1 \leqq X \leqq H^{2-\delta}$.

Proof. Observe that

$$
\psi(r, T, H)=\psi_{1}+\psi_{2}, \quad \text { where } \psi_{1}=\psi(r, T, H)-\psi\left(r^{\prime}, T, H\right)
$$

and $\psi_{2}=\psi\left(r^{\prime}, T, H\right)$. Next we note that $\psi_{2} H^{\delta r^{\prime} / 2}$ tends to zero (with the required fastness), as $r^{\prime}$ tends to infinity. Theorem 4 follows from the following observations.

$$
\begin{aligned}
\psi_{1} \leqq & \frac{1}{H} \int_{T}^{T+H}\left|\left(\mid G(1 / 2)+r^{\prime}+i t\right)-\sum_{n \leqq H} \frac{b_{n}}{n^{1 / 2+r^{\prime}+i t}}\right|^{2} X^{r^{\prime}} \\
& \left.-\left|G(1 / 2+r+i t)-\sum_{n \leqq H} \frac{b_{n}}{n^{1 / 2+r+i t}}\right|^{2} X^{r}\right) \mid d t .
\end{aligned}
$$

(2) In view of $\left.|| A\right|^{2}-|B|^{2}|\leqq| A^{2}-B^{2} \mid$, valid for any two complex numbers $A, B$, the integrand in (1) does not exceed

$$
\int_{\boldsymbol{r}}^{r^{\prime}}\left|\frac{d}{d u}\left(\left(G(1 / 2+u+i t)-\sum_{n \leqq H} \frac{b_{n}}{n^{1 / 2+u+i t}}\right)^{2} X^{u}\right)\right| d u .
$$

(3) We can treat $u$ as a complex variable, and if we write the integrand in (2) as $|(d / d u) \varphi(u)|$ with an obvious notation we see that

$$
\frac{d}{d u} \varphi(u)=\frac{1}{2 \pi i} \int_{R} \frac{\varphi(w) e^{(w-u)^{4 a+2}}}{(w-u)^{2}} d w,
$$

where $R$ is a rectangle containing $u$ in its interior, provided $\varphi(w)$ is regular inside $R$ and continuous on the boundary of $R$. Here $a$ is a positive integer constant (its largeness depending on how small $\eta$ is) and is at our disposal.

(4) We choose for $R$ the rectangle bounded by the lines $\operatorname{Re} w=0, \operatorname{Re} w=\infty$, $|\operatorname{Im} w|=H^{\eta}$ (note that $\varphi(u)$ tends to zero as $u$ tends to infinity). Here and in similar circumstances in future the phrase " $\operatorname{Re} w=\infty$ " refers to $\operatorname{Re} w$ being a constant which is large enough.

(5) When $|\operatorname{Im} w|=H^{\eta}$, the factor $\exp \left((w-u)^{4 a+2}\right)$ makes the contributions to the integral in (3) very small.

$$
\int_{\substack{\operatorname{Re} w=0 \\-\infty<\operatorname{Im} w<\infty}}\left|\frac{e^{(w-u)^{4 a+2}}}{(w-u)^{2}}\right||d w|=O\left(\frac{1}{u}\right),
$$

where the $O$-constant depends only on $a$. 
The observations (1) to (6) give an upper bound for $\psi_{1}$. To estimate $\psi_{2}$ we make the following observations.

$$
\begin{gathered}
\psi_{2} H^{\delta r^{\prime} / 2}=\frac{1}{H} \int_{T}^{T+H}\left|\varphi\left(r^{\prime}\right) H^{\delta r^{\prime} / 2}\right| d t, \\
\varphi\left(r^{\prime}\right) H^{\delta r^{\prime} / 2}=\frac{1}{2 \pi i} \int_{R_{1}} \frac{\varphi(w) H^{\delta w / 2} e^{\left(w-r^{\prime}\right)^{4 a+2}}}{w-r^{\prime}} d w,
\end{gathered}
$$

where $R_{1}$ is the rectangle bounded by the lines $\operatorname{Re} w=r, \operatorname{Re} w=\infty,|\operatorname{Im} w|=H^{\eta}$.

$$
\int_{\substack{\operatorname{Re} w=R \\-\infty<\operatorname{Im} w<\infty}}\left|\frac{e^{\left(w-r^{\prime}\right)^{4 a+2}}}{w-r^{\prime}}\right||d w|=O\left(\log \left(\frac{1}{r^{\prime}-r}\right)\right),
$$

where the $O$-constant depends only on $a$.

The observations (7), (8), (9) give an upper bound for $\psi_{2} H^{\delta r^{\prime} / 2}$ and this completes the proof of Theorem 4 with $\delta_{1}=\delta / 2$.

Theorem 5. Let $\sum_{n=1}^{\infty} d_{n}^{\prime} / n^{s}$ be absolutely convergent for $\sigma>1$, and let $G(s)=\left(\sum_{n=1}^{\infty} d_{n}^{\prime} / n^{s}\right)^{2}$ be analytic in $\sigma \geqq-1$ and $|t| \geqq 100$, and here satisfy the functional equation

$$
G(s)=\chi(s) G(1-s)
$$

and the uniform estimate $G(s)=O\left(\exp \left((\log |t|)^{100}\right)\right)$. Suppose further that the function $\chi(s)$ is analytic in the said region and that for some positive constant $k$

$$
\chi(s)=O\left(|t|^{k(1 / 2-\sigma)}\right), \quad(O \text {-constant }=1 \text { if } \sigma=1 / 2),
$$

where the $O$-constant depends only on $\sigma$ and in a continuous way. Next let

and for real $r$ define

$$
M_{2}(\sigma)=\frac{1}{H} \int_{T}^{T+H_{1}}\left|G(s)-\left(\sum_{n \leqq H} \frac{d_{n}^{\prime}}{n^{s}}\right)^{2}\right|_{s=\sigma+i t} d t, \quad\left(H_{1}>0\right),
$$

$$
\vartheta\left(r, T, H_{1}\right)=M_{2}(1 / 2+r) X^{r} \text {, where } X=H^{1-\delta} \quad \text { and }
$$

$\delta$ is a positive constant less than 1 . Then if $0<r_{1}<r$ and $0<\eta<1$, we have

$$
\begin{gathered}
|\vartheta(0, T, H)-\vartheta(r, T, H)|=O\left(\left(\vartheta\left(r, T-H^{\eta}, H+2 H^{\eta}\right)\left(\frac{T^{k}}{X^{2}}\right)^{r}+X^{-r} \sum_{n \leqq H} \frac{\left|d_{n}^{\prime}\right|^{2}}{n^{1-2 r}}\right) \frac{r_{1}}{r}\right. \\
+\min \left(\vartheta\left(0, T-H^{\eta}, H+2 H^{\eta}\right) \log \left(\frac{r}{r_{1}}\right),\left(\vartheta\left(r, T-H^{\eta}, H+2 H^{\eta}\right)\left(\frac{T^{k}}{X^{2}}\right)^{r}\right.\right. \\
\left.\left.\left.+X^{-r} \sum_{n \leqq H} \frac{\left|d_{n}^{\prime}\right|^{2}}{n^{1-2 r}}\right) \log \left(\frac{2 r}{r+r_{1}}\right)\right)\right),
\end{gathered}
$$

where the $O$-constant depends only on $\eta$ and $\delta$. 
Proof. We write

$$
\vartheta(0, T, H)-\vartheta(r, T, H)=\vartheta(0, T, H)-\vartheta\left(r_{1}, T, H\right)+\vartheta\left(r_{1}, T, H\right)-\vartheta(r, T, H)
$$

and proceed as in the proof of Theorem 4. (For both differences on the right we have to use the derivative of some function like $\varphi(u))$. In the case of the first difference we take the left side of the rectangle to be $\operatorname{Re} w=-r$. In the case of the second we take both the rectangles with sides $\operatorname{Re} w=0$ and $\operatorname{Re} w=-r$. On the side $\operatorname{Re} w=-r$ we use the functional equation for $G(s)$.

From Theorem 5 we deduce two corollaries which are in fact important enough to be called theorems.

Corollary 1 . Suppose $\sigma-1 / 2=O(1 / \log T)$. Then

$$
\frac{1}{H} \int_{T}^{T+H}|G(1 / 2+i t)| d t=O\left(\frac{1}{H} \int_{T-H^{\eta}}^{T+H+H^{\eta}}|G(\sigma+i t)| d t+\sum_{n \leqq H} \frac{\left|d_{n}^{\prime}\right|^{2}}{n}\right),
$$

and

$$
\frac{1}{H} \int_{T}^{T+H}|G(\sigma+i t)| d t=O\left(\frac{1}{H} \int_{T-H^{\eta}}^{T+H+H^{\eta}}|G(1 / 2+i t)| d t+\sum_{n \leqq H} \frac{\left|d_{n}^{\prime}\right|^{2}}{n}\right),
$$

where the $O$-constant depends only on $\eta$ and the $O$-constant in $O(1 / \log T)$.

Proof. It suffices to prove with $\sigma>1 / 2$ and then apply the functional equation. To prove the second part choose $r_{1}$ small but with $r / r_{1}$ bounded, and then take only the first term in $\min (\ldots, \ldots)$. To prove the first part choose $r_{1}$ such that $r_{1}$ nearly equal to $r$ and take only the second term in $\min (\ldots, \ldots)$.

Corollary 2. Let $0<k<2, k / 2<\lambda<1, H=T^{\lambda}$. Then for $l \geqq 0$, an integer, we have

$$
\frac{1}{H} \int_{T}^{T+H}\left|G^{(l)}(1 / 2+i t)\right| d t=O\left(\sum_{n \leqq H} \frac{\left|d_{n}^{\prime}\right|^{2}}{n}(\log H)^{l}\right),
$$

where the $O$-constant is independent of $T$.

Proof. It suffices to prove the corollary with $l=0$. (Cauchy's theorem makes possible the extension to any integer $l \geqq 0$ ). For this we note that $G(\sigma+i t)=$ $O_{\varepsilon}\left(t^{k / 4+\varepsilon}\right)$ (by the functional equation) holds for every $\varepsilon>0 \quad\left(O_{\varepsilon}\right.$ indicates that the constant depends on $\varepsilon)$ and $\sigma-1 / 2=O(1 / \log T)$ and hence $(1 / H) \int|G(\sigma+i t)| d t$, the integral being over any $t$ interval of length $H^{\eta}$ contained in $(T / 2,2 T)$ is $o(1)$. Hence by Corollary 1 , the integrals $(1 / H) \int_{T}^{T+H}|G(\sigma+i t)| d t$ are of the same order if Corollary 2 is false with $l=0$, provided $\sigma-1 / 2=O(1 / \log T)$. We now denote the last integral with $\sigma=1 / 2$ by I, put $X=H^{1-\delta}$ and $H^{1-2 \delta}$ in Theorem 5 and subtract. We get, letting $r=K / \log T$, where $K$ is a large positive constant,

$$
\left|\mathrm{I}\left(H^{r(1-2 \delta)}-H^{r(1-\delta)}\right)\right|=O\left(\mathrm{I}\left(\frac{T^{k}}{H^{1-2 \delta}}\right)^{r} \frac{r_{1}}{r}+\mathrm{I}\left(\frac{T^{k}}{H^{1-2 \delta}}\right)^{r} \log \frac{2 r}{r+r_{1}}\right)
$$


by taking the second term in $\min (\ldots, \ldots)$. Let us fix $r_{1}$ to be $r / 2$. This gives

$$
1=O\left(\left(\frac{T^{k}}{H^{2-3 \delta}}\right)^{r}\right)
$$

which is a contradiction (unless $\lambda \leqq k / 2$; note we have assumed that $k / 2<\lambda<1$ ).

Corollary 2 trivially gives Theorem 2. However, we show in Part $\mathrm{C}$ that the more general proof in Part $\mathrm{B}$ also gives this and more.

\section{Part B}

In the remainder of this paper $F(s)$ has a meaning other than what it had before. $F(s)$ shall be represented in $\sigma>1$ by the square of an absolutely convergent Dirichlet series $\sum_{n=1}^{\infty} d_{n} / n^{s}$. It shall be regular in $\sigma>1 / 2,|t| \geqq 10$ and continuous in $\sigma \geqq 1 / 2$, $|t| \geqq 10$. We now describe the next restriction. There exist a finite set $\left\{f_{j}(s)\right\}$ of functions analytic in $|t| \geqq 10$, each represented in $\sigma>1$ by an absolutely convergent Dirichlet series. Next each of the functions $f_{j}(s)$ is required to satisfy a functional equation

$$
f_{j}(s)=\chi_{j}(s) f_{j}(1-s)
$$

where $\varkappa_{j}(s)$ are analytic in $|t| \geqq 10$, and further $\varkappa_{j}(s)=O\left(\mid t^{g_{j}(1 / 2-\sigma)}\right)$, where the $O$-constant is 1 if $\sigma=1 / 2$ and depends only on $\sigma$ and in a continuous way. (It can also depend on $j$ ). With positive constant $k_{j}$ we define the function $\left(f_{j}(s)\right)^{k_{j}}$ as the one which results from analytic continuation starting from a right half plane where $f_{j}(s) \neq 0$, along all possible lines parallel to the real axis. We impose on $f_{j}(s)$ the further condition that in $\sigma \geqq-1,|t| \geqq 10$, it should satisfy $f_{j}(s)=O\left(\exp \left((\log |t|)^{100}\right)\right)$ where the $O$-constant is independent of $t$. The restriction on $F(s)$ can now be stated as

$$
F(s)=\prod_{j}\left(f_{j}(s)\right)^{k_{j}}
$$

for a suitable finite set of functions $f_{j}(s)$ and constants $k_{j}>0$. Now $F(s)=$ $\varkappa(s) F(1-s)$, where neither $F(s)$ nor $\chi(s)$ need be single valued but $\chi(s)=O\left(|t|^{k(1-2 \sigma) / 2}\right)$ with $k=\sum g_{j} k_{j}$, and the constant implied by the $O$-symbol is 1 if $\sigma=1 / 2$ and depends only on $\sigma$ and in a continuous way.

We are now in a position to state

Theorem 6. Let $F(s)=\left(\sum_{n=1}^{\infty} d_{n} / n^{s}\right)^{2}$ in $\sigma>1$, and let $0<k<4, k / 4<\lambda<1$, $H=T^{\lambda}$. Then we have

$$
\frac{1}{H} \int_{T}^{T+H}\left|F^{(l)}(1 / 2+i t)\right| d t=O\left(\left(\sum_{n \leqq H} \frac{\left|d_{n}\right|^{2}}{n}\right)(\log T)^{l}\right),
$$

provided $\left(d^{l} / d s^{l}\right)(F(s))^{1 / 2}$ is analytic in $\sigma>1 / 2,|t| \geqq 10$, and continuous in $\sigma \geqq 1 / 2$ and $|t| \geqq 10$. (In fact the validity of the last two conditions in $T-H / 2 \leqq t \leqq T+3 H / 2$ is enough for the validity of the theorem.) 
We prove this theorem by a series of lemmas of which 1, 2 and 3 are standard. They are to be found in Titchmarsh's book (see p. $180, \S 5.65 ;$ p. $125, \S 3.61$; and p. $311, \S 9.623$ of [5]) on the theory of functions. We give the proofs only for the sake of completeness.

Lemma 1. We have $f_{j}(\sigma+i t)=O_{\varepsilon}\left(|t|^{g_{j}((1-\sigma) / 2)+\varepsilon}\right)$, where $0 \leqq \sigma \leqq 1,|t| \geqq 10$ and the $O$-constant depends only on $\varepsilon>0$, which is arbitrary.

Proof. We have $f_{j}(1+\varepsilon+i t)=O_{\varepsilon}(1)$ and $f_{j}(-\varepsilon+i t)=O_{\varepsilon}\left(|t|^{\left(g_{j}+2 \varepsilon\right) / 2}\right)$. Next with $s=\sigma+i t$ we have

$$
f_{j}(s)=\frac{1}{2 \pi i} \int_{R_{2}} f_{j}(w)|t|^{g_{j}((w-s) / 2)} e^{(w-s) 20} \frac{d w}{w-s},
$$

where $R_{\mathbf{2}}$ is the rectangle bounded by the sides $\operatorname{Re} w=-\varepsilon, 1+\varepsilon,|\operatorname{Im} w|=\log |t|$. Rough estimates now lead to the lemma.

Lemma 2. Let $f(z)$ be analytic in $|z| \leqq r$ and let @ run over all the zeros of $f(z)$ in this disc. Then

$$
\log \left(|f(0)| \prod_{\varrho} \frac{r}{|\varrho|}\right)=\int_{0}^{1} \log \left|f\left(r e^{2 \pi i \theta}\right)\right| d \theta .
$$

Proof. If there are no zeros, $\log f(z)$ can be expanded as a power series (in $|z| \leqq r)$ in $z$, which is convergent. Writing $z=r e^{2 \pi i \theta}$ and integrating term by term, we get

$$
\log f(0)=\int_{0}^{1} \log f\left(r e^{2 \pi i \theta}\right) d \theta
$$

Taking the real parts on both sides we get the result required. But if $f(z)$ has zeros in $|z| \leqq r$, we replace $f(z)$ by

$$
f(z) \prod_{\varrho}\left\{\left(\frac{r^{2}-z \bar{\varrho}}{r^{2}(1-z / \varrho)}\right) \frac{r}{\varrho}\right\} .
$$

Notice that on $|z|=r$, i.e. $z \bar{z}=r^{2}$ (bar denotes complex conjugation), this function has the same absolute value as $f(z)$. This completes the proof of the lemma.

Lemma 3. Let $\varphi_{0}(t)$ be a continuous function defined in $a \leqq t \leqq b$. If $\varphi_{0}(t)$ is non-negative throughout this interval, then

$$
\frac{1}{b-a} \int_{a}^{b} \log \varphi_{0}(t) d t \leqq \log \left(\frac{1}{b-a} \varphi_{0}(t) d t\right) .
$$

Proof. Let $a=x_{0}<x_{1}<x_{2} \ldots<x_{n}=b$. Then we have

$$
\left(\varphi_{0}\left(x_{1}\right) \varphi_{0}\left(x_{2}\right) \ldots \varphi_{0}\left(x_{n}\right)\right)^{1 / n} \leqq \frac{\varphi_{0}\left(x_{1}\right)+\varphi_{0}\left(x_{2}\right)+\ldots+\varphi_{0}\left(x_{n}\right)}{n}
$$


ie.

$$
\sum_{v=1}^{n}\left(\log \left(\varphi_{0}\left(x_{v}\right)\right)\left(\frac{1}{n}\right)\right) \leqq \log \left(\sum_{v=1}^{n}\left(\varphi_{0}\left(x_{v}\right)\left(\frac{1}{n}\right)\right)\right) .
$$

This proves the lemma by the obvious limiting process.

Lemma 4. If $s=\sigma+i$ is any complex number with $|t| \geqq 100$ and $X_{1}>0$, then

$$
\left.|F(s)| \leqq \int_{0}^{1}\left|F\left(s+r e^{2 \pi i \theta}\right)\right| X_{1}^{r \cos (2 \pi \theta)} d \theta \quad \text { (we assume } 0<r \leqq 2\right) .
$$

Proof. Apply Lemma 2 with $f(z)=f_{1}(s+z), f_{2}(s+z), \ldots$ and $f(z)=X_{1}^{z}$ and take an appropriate linear combination. Denoting by $\varrho_{j}$ the zeros of $f_{j}(s+z)$ in $|z| \leqq r$, we get

$$
\log \left\{|F(s)| \prod_{j} \prod_{\varrho_{j}}\left(\frac{r}{\left|\varrho_{j}\right|}\right)^{k_{j}}\right\}=\int_{0}^{1} \log \left(\left|F\left(s+r e^{2 \pi i \theta}\right)\right| X_{1}^{r \cos (2 \pi \theta)}\right) d \theta .
$$

Applying Lemma 3 we get

$$
|F(s)|\left(\prod_{j}\left(\prod_{\varrho_{j}}\left(\frac{r}{\left|\varrho_{j}\right|}\right)^{k_{j}}\right)\right) \leqq \int_{0}^{1}\left|F\left(s+r e^{2 \pi i \theta}\right)\right| X_{1}^{r \cos (2 \pi \theta)} d \theta .
$$

This completes the proof of Lemma 4 since here the LHS is $\geqq|F(s)|$.

Lemma 5. Let $s=1 / 2+i t$ and $T-H^{\eta} \leqq t \leqq T+H+H^{\eta}$, where $\eta$ is a constant satisfying $0<\eta<1$. Then

(we assume $0<r \leqq 2$ ).

$$
|F(s)| \leqq \int_{0}^{1}\left|F\left(s+r e^{2 \pi i \theta}\right)\right| T^{(k r \cos (2 \pi \theta)) / 2} d \theta
$$

Proof. Follows from Lemma 4 if we put $\sigma=1 / 2$ and take $X_{1}=T^{k / 2}$.

Lemma 6. In the notation of Lemma 5, we have

$$
|F(s)|=O\left(\int_{0}^{1}\left\{\left(\left|F\left(s+r x+i r \sqrt{1-x^{2}}\right)\right|+\left|F\left(s+r x-i r \sqrt{1-x^{2}}\right)\right|\right) T^{k r x / 2}\right\} \frac{d x}{\sqrt{1-x^{2}}}\right) .
$$

Here $0<r \leqq 2$, and the $O$-constant is independent of $r$ and $s$.

Proof. Follows if we break up the interval 0, 1 into four parts depending on the four quadrants in which $\mathrm{e}^{2 \pi i \theta}$ lies, use the functional equation for

$$
|F(s-r \cos (2 \pi \theta) \pm i r \sin (2 \pi \theta))|
$$

and then make the substitution $x=\cos (2 \pi \theta)$.

Lemma 7. In the notation of Lemma 5, we have

$$
|F(s)|^{1 / 2}=O\left(\int_{0}^{1}\left\{\left(\left|F\left(s+r x+i r \sqrt{1-x^{2}}\right)\right|^{1 / 2}+\left|F\left(s+r x-i r \sqrt{1-x^{2}}\right)\right|^{1 / 2}\right) T^{k r x / 4}\right\} \frac{d x}{\sqrt{1-x^{2}}}\right) .
$$

Here $0<r \leqq 2$ and the $O$-constant is independent of $r$ and $s$. 
Proof. Prove Lemma 4 with $|F(s)|$ replaced by $|F(s)|^{1 / 2}$ and with $X_{1}$ replaced by $T^{k / 4}$. Next proceed as in Lemmas 5 and 6 .

Lemma 8 . Let $E=\sum_{n \leqq H}\left|d_{n}\right|^{2} / n$,

$$
I^{*}(1 / 2+r)=\frac{1}{H} \int_{T}^{T+H}\left|(F(1 / 2+r+i t))^{1 / 2}-\sum_{n \leqq H} \frac{d_{n}}{n^{1 / 2+r+i t}}\right|^{2} d t, \quad(r>0),
$$

and $J(r)=I^{*}(1 / 2+r) T^{k r / 2}$. Let us agree to choose that value of the square root which makes $(F(s))^{1 / 2}=\sum_{n=1}^{\infty} d_{n} / n^{s}$ when real part of $s$ is $>1$. Then for $0<r \leqq 2$, we have

$$
J(0)=O\left(\int_{0}^{1} J(r x) \frac{d x}{\sqrt{1-x}}+E T^{k r / 2}\right) .
$$

Proof. In Lemma 7 use $|F(\ldots)|^{1 / 2} \leqq\left|(F(\ldots))^{1 / 2}-\sum_{n \leqq H} d_{n} / n \cdots\right|+\left|\sum_{n \leqq H} d_{n} / n \cdots\right|$, on the right side of the inequality. Next apply Hölder's inequality to obtain an upper bound for $(1 / H) \int_{T}^{T+H}|F(1 / 2+i t)| d t$. The expressions which bound this integral involve not $I^{*}(r x)$ but things which differ from $(1 / H) \int_{T}^{T+H}|\ldots| d t$ by amounts $(1 / H) \int|\ldots| d t$, where the integral is over an interval of length $H^{\eta}$ contained in $T-H / 2$ and $T+3 H / 2$. Since $\eta$ is arbitrary, it follows from Lemma 9 that we can replace (with negligible loss) the new limits of integration back by $T$, $T+H$. Hence Lemma 8 follows from Lemma 7 and Lemma 9 (to follow) and the observation that in $0 \leqq x \leqq 1,1 / \sqrt{1-x^{2}}=O(1 / \sqrt{1-x})$.

Lemma 9. In $|t| \geqq 10$ and $0 \leqq \sigma \leqq 1$ we have

$$
|F(\sigma+i t)|=O_{\varepsilon}\left(|t|^{k(1-\sigma) / 2+\varepsilon}\right)
$$

where $\varepsilon>0$ is arbitrary and the $O$-constant is independent of $\sigma$ and $t$ but depends on $\varepsilon$.

Proof. Lemma 9 follows as an immediate corollary to Lemma 1.

Lem ma 10. There exists a positive constant $\delta>0$, depending only on $\lambda$, such that

$$
\int_{0}^{1} J(r x) \frac{d x}{\sqrt{1-x}}=O\left(\frac{J(0)}{r \log T}+J_{1}(r)\right),
$$

where $J_{1}(r)=J(r) T^{\delta r}$.

Proof. L.H.S $=\int_{0}^{1} J_{1}(r x) T^{-\delta r x} d x / \sqrt{1-x}$ and the error in replacing $J_{1}(r x)$ by $J_{1}(r)$ is $O\left(J(0) \int_{0}^{1} T^{-\delta r x} d x / \sqrt{1-x}\right.$ ), provided $\delta$ is small (this follows by an argument similar to a step in the proof of Theorem 4). This proves Lemma 10.

In Lemmas 11 to 16 (below) our main task is the estimation of $J_{1}(r)$. The method followed is an extension of the method of proof of Theorem 4 and our results may be stated as a general Theorem but we do not do it. Note that $J(0)=J_{1}(0)$. 
Lemma 11. For any fixed positive number $v$, put $r_{n}=r(1+v)^{n}(n=0,1,2,3, \ldots)$. Suppose that $0<v \leqq 1 / 2$. Then for any positive constant $\delta$ we have

$$
\frac{J_{1}(r)}{T^{\delta r}}=\sum_{n=1}^{\infty} u_{n}, \text { where } u_{n}=\frac{J_{1}\left(r_{n-1}\right)}{T^{\delta r_{n-1}}}-\frac{J_{1}\left(r_{n}\right)}{T^{\delta r_{n}}},
$$

provided that $J_{1}\left(r_{n}\right) T^{-\delta r_{n}}$ tends to zero as $n$ tends to infinity.

Proof. Trivial.

Lemma 12. We have

$$
J_{1}(r)=O\left(v T^{\delta r} J(0)+v J_{1}(r)+v \sum_{n=1}^{\infty} \frac{J_{1}\left(r_{n}\right)}{T^{\delta\left(r_{n}-r_{0}\right)}}\right),
$$

where the $O$-constant is independent of $v$ and $r$.

Proof. By an argument similar to a step in the proof of Theorem 4 we have

$$
u_{1}=O(v J(0)), \quad u_{2}=O\left(v \frac{J_{1}\left(r_{0}\right)}{T^{\delta r_{0}}}\right), \quad u_{3}=O\left(v \frac{J_{1}\left(r_{1}\right)}{T^{\delta r_{1}}}\right), \quad u_{4}=O\left(v \frac{J_{1}\left(r_{2}\right)}{T^{\delta r_{2}}}\right), \ldots
$$

and so on. Hence

$$
\frac{J_{1}(r)}{T^{\delta r}}=O\left(v J(0)+v \frac{J_{1}(r)}{T^{\delta r}}+v \sum_{n=1}^{\infty} \frac{J_{1}\left(r_{n}\right)}{T^{\delta r_{n}}}\right),
$$

and this gives the lemma.

Lemma 13. We have

$$
J_{1}(r)=O\left(v T^{\delta r} J(0)+v J_{1}(r)+v \sum_{n=1}^{\infty} \frac{J_{1}(r)+n v J(0)}{T^{\delta n r v}}\right) .
$$

Proof. By arguments similar to a step in the proof of Theorem 4, it follows that $J_{1}\left(r_{n}\right)=J_{1}(r)+O(v n J(0))$, and this proves Lemma 13 .

Lemma 14. We have

$$
J_{1}(r)=O\left(v T^{\delta r} J(0)+v J_{1}(r)+\frac{v J_{1}(r)}{T^{\delta r v}-1}+\frac{v^{2} T^{\delta r v} J(0)}{\left(T^{\delta r v}-1\right)^{2}}\right) .
$$

Proof. Follows from Lemma 13 if we use

$$
\sum_{n=1}^{\infty} x^{n}=\frac{x}{1-x} \text { and } \sum_{n=1}^{\infty} n x^{n}=\frac{x}{(1-x)^{2}} \quad \text { both valid in } 0<x<1
$$

Lemma 15. There exists a positive constant $\delta$ such that

$$
J_{1}(r)=O\left(T^{-\delta r}\left(J(0)+J_{1}(r)\right)+\frac{J_{1}(r)}{r \log T}+\frac{J(0)}{(r \log T)^{2}}\right) .
$$

Proof. In Lemma 14 choose $v=T^{-2 \delta r}$ and note that $1 /\left(T^{\delta r v}-1\right)$ and $T^{\delta r v} /\left(T^{\delta r v}-1\right)^{2}$ are $O(1)$ if $T^{\delta r v} \geqq 10$, but $O(1 /(v r \log T))$ and $O\left(1 /(v r \log T)^{2}\right)$ otherwise. This proves the lemma. 
Lemma 16. We have

$$
J_{1}(r)=O\left(\frac{J(0)}{(r \log T)^{2}}\right)+O\left(\frac{J_{1}(r)}{r \log T}\right) .
$$

Remark. Lemma 16 follows from Lemma 15. Note that Lemmas 11 to 16 (in fact also Lemma 10) are independent of the rest of the paper though they are useful in the proof of Theorem 6 as will be seen. These lemmas require that $k / 4<\lambda<1$ and $\delta$ depends only on $\lambda-k / 4$.

Lemma 17. If $r \log T$ exceeds a constant $C$, then

$$
\int_{0}^{1} J(r x) \frac{d x}{\sqrt{1-x}}=O\left(\frac{J(0)}{r \log T}\right) .
$$

Proof. If $r \log T$ is large enough (say $\geqq C$ ), then the second $O$-term in Lemma 16 is negligible and so $J_{1}(r)=O\left(J(0) /(r \log T)^{2}\right)$. Hence Lemma 17 follows from Lemma 10.

Lemma 18. If $r \log T$ exceeds a large constant $C$ (depending on $\lambda-k / 4$ ), then

and so

$$
J(0)=O\left(\frac{J(0)}{r \log T}\right)+O\left(T^{k r / 2} \sum_{n \leqq H} \frac{\left|d_{n}\right|^{2}}{n}\right),
$$

$$
J(0)=O\left(T^{k r / 2} \sum_{n \leqq H} \frac{\left|d_{n}\right|^{2}}{n}\right) .
$$

Proof. Apply Lemmas 8 and 17.

Remarks concluding Part B. The second statement of Lemma 18 proves Theorem 6 with $l=0$ (we have only to take $r=2 C / \log T$ ). The case $l>0$ can be proved by a modification of our method. From Lemmas 16, 18 and 8 it follows that $I^{*}(1 / 2+r)=O(E)$ for all $r$ lying between $2 C / \log T$ and $4 C / \log T$. A simple application of Cauchy's theorem gives (by use of this result)

$$
\frac{1}{H} \int_{T}^{T+H}\left|\frac{d^{l}}{d s^{l}} F(s)\right| d t=O\left(E(\log T)^{l}\right)
$$

where $\sigma=3 C / \log T+1 / 2$. But then the integral on the left differs from the corresponding integral with $\sigma=1 / 2+1 / \log T$ by an amount $O\left(E(\log T)^{l}\right)$ by an argument similar to a step in the proof of Theorem 4 (we have to use $I^{*}(1 / 2)=J(0)=$ $O(E)$ ). These remarks prove for instance that, for all integers $l \geqq 0$,

$$
\frac{1}{H} \int_{T}^{T+H}\left|F^{(l)}\left(\frac{1}{2}+\frac{1}{\log T}+i t\right)\right| d t=O\left(\left(\sum_{n \leqq H} \frac{\left|d_{n}\right|^{2}}{n}\right)(\log T)^{l}\right) .
$$




\section{Part C}

Theorem 7. Let $F(s)$ be represented in $\sigma>1$ by $\left(\sum_{n=1}^{\infty} d_{n} / n^{s}\right)^{2}$ and let the notation of Part $B$ before Theorem 6 be in force. Let $0<k<2, k / 2<\lambda<1, H=T^{\lambda}$. Then we have

$$
\frac{1}{H} \int_{T}^{T+H}\left|F^{(l)}(1 / 2+i t)\right| d t=O\left(\left(\sum_{n \leqq H} \frac{\left|d_{n}\right|^{2}}{n}\right)(\log T)^{l}\right),
$$

provided $F^{(l)}(s)$ is analytic in $\sigma>1 / 2,|t| \geqq 10$ and is continuous in $\sigma \geqq 1 / 2,|t| \geqq 10$. (In fact the validity of the conditions are enough in $T-H / 2 \leqq t \leqq T+2 H$ for the validity of the result asserted above.) Also we have for all non-negative integers $l$

$$
\frac{1}{H} \int_{T}^{T+H}\left|F^{(l)}\left(\frac{1}{2}+\frac{1}{\log T}+i t\right)\right| d t=O\left(\left(\sum_{n \leqq H} \frac{\left|d_{n}\right|^{2}}{n}\right)(\log T)^{l}\right) .
$$

Proof. We argue as in the proof of Theorem 6. Instead of Lemma 7 we apply Lemma 6 and instead of Lemma 8 we have a similar lemma with $I^{*}(1 / 2+r)$ replaced by

$$
\frac{1}{H} \int_{T}^{T+H}\left|F(1 / 2+r+i t)-\left(\sum_{n \leqq H} \frac{d_{n}}{n^{1 / 2+r+i t}}\right)^{2}\right| d t
$$

and this necessitates the condition $k / 2<\lambda<1$ in the rest of the argument. These considerations complete the proof of Theorem 7 .

We note a curious corollary to Theorem 7 . Let $\zeta_{K}(s)$ (we may also consider $L$-series etc.) be the Dedekind zeta function of an algebraic number field $K$ of degree d. Let $1 / 2<\lambda<1$ and $H=T^{\lambda}$. Then

$$
\frac{1}{H} \int_{T}^{T+H}\left|\zeta_{K}(1 / 2+i t)\right|^{1 / d} d t=O\left(\sum_{n \leqq H} \frac{\left|d_{n}\right|^{2}}{n}\right),
$$

where $\left(\zeta_{K}(s)\right)^{1 /(2 d)}=\sum_{n=1}^{\infty} d_{n} / h^{s}$ in $\sigma>1$, provided only that $\left(\zeta_{K}(s)\right)^{1 / d}$ is regular in $\sigma>1 / 2, t \geqq 1$. 


\section{References}

[1] RamachandRa, K.: Some remarks on the mean value of the Riemann zeta-function and other Dirichlet series - I. - Hardy-Ramanujan Journal, Vol. 1, 1978, 1-15.

[2] RamachandRa, K.: Some remarks on the mean value of the Riemann zeta-function and other Dirichlet series - II. - Hardy-Ramanujan Journal, Vol. 3, 1980, 1-24.

[3] Ramachandra, K.: Some remarks on a theorem of Montgomery and Vaughan. - J. Number Theory II, 1979, 465-471.

[4] Titchmarsh, E. C.: The theory of the Riemann zeta-function. - Oxford, 1951.

[5] Trtchmarsh, E. C.: The theory of functions. - Oxford, 1952.

Tata Institute of Fundamental Research

School of Mathematics

Homi, Bhabha Road

Bombay 400005

India

Received 3 May 1979 